\title{
Research on Propeller Characteristics of Tip Induced Loss
}

\author{
Yang Song ${ }^{1,}$, , Peng Shan ${ }^{2, b}$ \\ ${ }^{1}$ School of Jet Propulsion, Beihang University, Beijing 100191, China; \\ ${ }^{2}$ School of Jet Propulsion, Beihang University, Beijing 100191, China. \\ asonglike163@163.com
}

Keywords: propeller, prop fan, tip induced loss, loss characteristic.

\begin{abstract}
In the design process of modern advanced propeller or prop fan, tip induced loss has been the foremost cause of power cost and its natural of difficulty in quantization also causes the deviation of power on the design point. Based on the former research and combined BEM method with the Tip-Loss Factor, this paper analyzed two different cases which had determined geometries. Then, we got the characteristics of the two on the tip induced loss and made some quantitative estimates on induced power loss. The results showed that the propeller tip induced loss characteristics was not only related to the actual geometric distribution, but also had the correlation with the specific operational conditions, especially advanced ratio and pitch angle, which had the most obvious impact on this loss characteristics. Finally, this study illustrates that this manner adopted can be used for the design, experiment and theoretical analysis of actual propeller under certain conditions.
\end{abstract}

\section{Introduction}

In all kinds of aviation systems at present, advance propeller/prop fan ensures higher propulsive efficiency and low fuel consumption characteristics at the same time. Its advantages have aroused great interest in all countries especially in the background of increasingly scarce oil resources [1]. Compared with the traditional high bypass ratio turbofan engine, efficiency of turboprop propulsion system is about 20\% higher under Mach 0.8 and about 35\% [2] higher under Mach 0.7 in cruise state. Therefore, it is one of the major trends in the development of large and medium sized air transport in next few decades.

Compared with the design of traditional low-speed propeller, compression loss from the Mach effect is more obvious because of higher flight speed for advanced propeller. When blade aerodynamic force increases, the space of induced velocity field is changed drastically under certain conditions and then causes loss of kinetic energy from the air through the rotor plane. Usually, we call this Induced Loss. The loss is mainly manifested in the increase of extra power consumption.

Due to the gradual improvement of propeller design level, profile loss has been reduced and total loss is mainly concentrated in the hub and tip. The hub section is more thick because of structure requirement and the loss from hub-blade interference is unpredictable. Generally, its power loss is updated by designer themselves in the course of study. For blade section, tip vortex loss is about $25 \%$ of total loss including the movement of tip vortex and interaction between vortex and wake. Propeller tip induced loss, largely corresponding to the various losses associated with the tip vortex. Although there have been a lot of research on the development process of tip flow field, strong three-dimensional effect and unsteady turbulent space-time function of the physical nature make it hard to quantify the details of the vortex development or loss mechanism. Perhaps, there is no good method to reduce $t$ tip induced loss especially for the study of advanced propeller or prop fan. But if we can estimate loss percentage for a certain propeller, it must be able to provide some guidance for the theory, design and power calculation. This is also the purpose of this paper.

Most propeller theory can be used to calculate the induced velocity field and the difference among them lies in calculation precision and cost of the resources. From the early momentum theory, blade element theory to modern 3D compressible lifting line method, lift surface theory and CFD method, the accuracy of calculation in rotor disk is improved gradually. Determined by the complex turbulent 
characteristics of the vortex itself, high precision tip induced loss calculation has not much progress. Usually, we either ignore the loss of tip vortex which can be corrected by experiment later or use tip loss factor [3].

Based on the assumption of infinite blade, Betz was the first one to derive the optimal circulation distribution for a given propeller in the case of minimum power loss. On account of the work before, corresponding expressions was derived for the finite number of blades by Prandtl. He imported a factor to make optimal circulation from Betz at tip section equal to zero and this factor was defined as tip loss factor. After this, Goldstein derived the exact solution for propeller with a finite number of blades, solving the Poisson equation to make full use of the two-periodic spiral surface. Once the potential distribution in far field wake and tip speed ratio was known, the circulation and the performance parameters on a given radius point could be obtained.

As mentioned before, the calculation method of propeller induced velocity field has been quite mature and there are some methods to compute the distribution of tip loss factor along radius. But it is rare to combine these two fields for the quantitative analysis of tip induced loss. Thus, this paper will adopt BEM (Blade Element Momentum) method with tip loss factor to analyze the influence on performance parameters of propeller from tip induced loss. In the view of propeller similar theory, this result will provide necessary theoretical guidance for the experiment of advanced propeller and design of overall performance parameters without doubt.

\section{The Choice of Method for Induced Loss Calculation}

The method for induced loss analysis contains the calculation of induced velocity and the choice of tip loss factor. For this paper, appropriate physical model and related calculation methods are considered as follows.

(1) Sufficient accuracy to meet the requirements of the analysis of propeller performance parameters;

(2) Simplicity and effectiveness. The first one makes sure that process of obtaining the geometric data of propeller is simple and direct. The second one ensures faster convergence speed and less resource consumption for engineering.

While the accuracy of this method is slightly reduced, it's enough for the usual design state of propeller [4]. It has a good prediction of characteristics of local section of blade [5]. It is particularly suitable for the requirement of rapid verification on experimental design results in engineering. Compared with the calculation method of 3D performance, input data format required by the BEM method is quite simple.

Although tip loss factor is assumed to apply only to a limited number of blades and light-load propeller [3], NACA early report [6] on the experimental study about single-rotating propeller with different number of blades showed that experimental results has good agreement with the calculations. Consequently, tip loss factor is accurate enough for the study on actual performance of propeller.

Based on the general expression of Prandtl tip loss factor, Glauert [7] made some additional simplification and derivation due to the nature of tip flow which is difficult to quantify. These steps enhanced physical understanding of tip induced loss and ensured the effectiveness in the calculation. Because of avoiding the complexity from Goldstein exact solution, the factor has been applied in the most of literatures. It's also used in this paper and its form is as follows:

$$
F=\frac{2}{\pi} \cos ^{-1} \exp \left[-\frac{B}{2}\left(\frac{\lambda}{\lambda_{r}}-1\right) \frac{1}{\sin \varphi}\right]
$$

$\mathrm{B}$ is the number of blades. $\lambda, \lambda_{r}, \varphi$ is design advanced ratio, local advanced ratio and local flow angle.

In conclusion, the BEM method combined with appropriate loss factor will be use here to do some deep research on tip induced loss. On one hand, conventional BEM method was applied to design and 
optimization of blade and there is little research on propeller tip induced loss. On the other hand, the similar map about propeller tip loss will be an effective format for further application.

In addition, due to the nature of 2D BEM method, effectiveness of the results is entirely dependent on the given lift-drag characteristics. Because tip loss factor is a kind of idealized model, there are some reasons to believe that the estimation from this work can be a lower limit for propeller design and experiment. It is still unable to quantify the upper limit of tip induced loss due to extreme change of turbulence. Detailed derivation of BEM method can be found in the literature [3], which we can use for the study on propeller overall performance.

\section{Analysis on fixed pitch propeller}

Reference section of 0.70 radius of this fixed pitch propeller is selected from literature [7]. Generally, propeller design theory needs to ensure this section is in a small angle of attack. Consequently, this angle is chosen as 2 . The design advanced ratio is chosen as 0.227 . The airfoil chosen is consistent with the literature.

First of all, the result about induced velocity filed in rotor plane is need to be verified. Compared with the data provided by Glauert, it is shown in Figure 1.

Figure 1 displays clearly that the results form BEM method used in this paper is in good agreement with the data, particularly when the angle of attack of the reference section is less than 8 . For induced axial factor, the calculation results under small angle of attack are closer to the data and slightly higher at high angle of attack. For induced tangential factor, the results are consistent with the data. The accuracy of BEM method is reduced when angle of attack is further increased and more than 8. In this case, the axial factor has been significantly deviated from the data under high angle. This is because when the angle of attack is too large, the flow on the reference section has been separated. Now propeller is under an unstable condition and difficult to work for a long time.

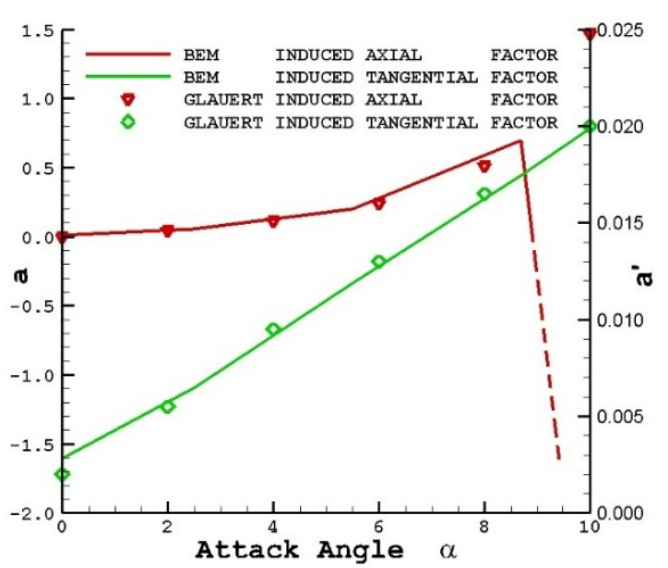

Fig. 1 Comparison on propeller induced velocity field between literature data and BEM method in this paper

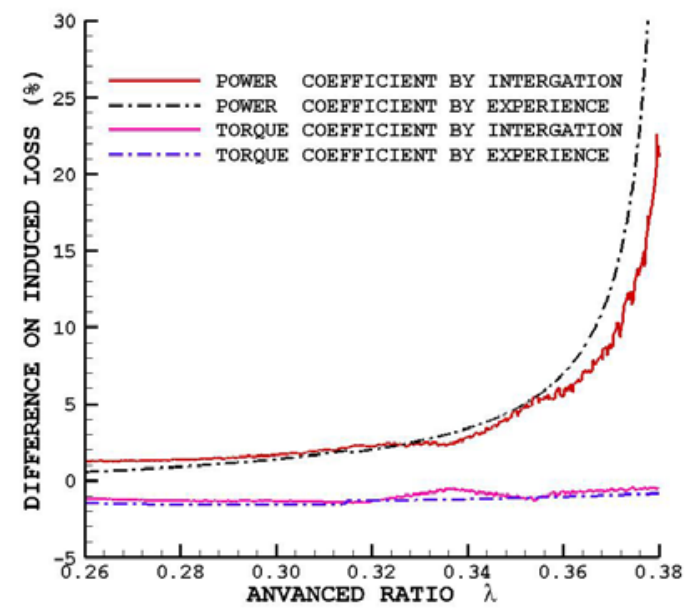

Fig. 2 Comparison on propeller tip induced loss Characteristic between experience formulae from literature [7] and current method

On one hand, unless there is a loss of stability in the state corresponding to the airfoil lift-drag characteristic, the difference is inevitable from limitation of the original data. The information used in the literature are slightly inadequate. On the other hand, different numerical methods about positive and inverse problem may also be one of the reasons for the deviation. In summary, the accuracy of the BEM method used in this paper is enough for requirements of the calculation of propeller performance parameters under actual conditions. 
Further analysis of this propeller, its characteristics of tip induced loss is shown in Figure 2. This feature is the influence of tip loss caused by power coefficient and torque coefficient. In full range of working states, the results from calculation and empirical formula are basically the same, but the specific value is slightly different. The calculated number of power coefficient is higher under the circumstances of low advanced ratio. The difference between the two is less than $1 \%$. With the gradual increase in advanced ratio, this difference is also increased. When advanced ratio is 0.37 , the difference reached $4 \%$. For the torque coefficient, the two things are a little different.

We can also find that when the advanced ratio is small, the power loss caused by the tip is not serious. With the advanced ratio rises, the power loss is gradually increasing. The loss is more than $10 \%$ in some specific conditions and even reached $20 \%$. Tip induced loss does large effect on the performance of the propeller, which is consistent with the qualitative understanding of propeller loss.

\section{Analysis on variable pitch propeller}

The analysis is based on a variable pitch propeller from literature [6]. The propeller has 6 blades and its diameter is $3.08 \mathrm{~m}$. Its reference section is at the 0.75 radius. The maximum efficiency is existed in when the pitch angle is 45 . The attack of angle of this case on reference section is 2 . The design advanced ratio is 0.700. The airfoil is CLARK-Y which is same as the literature. The geometric parameters of this propeller are shown in Figure 3. The characteristics of tip induced loss is shown in Figure 4.

From the picture, we can find that with increase of pitch angle, the tip induced loss in the gradually reduced for this propeller. That means influence on power coefficient and torque coefficient is decreased. When the pitch is given, advance ratio increases, the tip induced loss increases. It is worth noting that, for this propeller, when pitch angle is 20 under large advanced ratio conditions, induced loss of power has reached the maximum, more than $4 \%$. It's also can be found from Figure 3 that tip induced loss of properties change dramatically in the settings of small pitch angle. These two situations are due to the fact that local airfoil has a negative angle of attack. Loss is not so obvious in some cases of large pitch angle. This is because the pitch rises much closer to the design state. Generally speaking, the optimal pitch angle of high-speed propeller is about 40. The loss from flow separation and tip induced has minimum value.

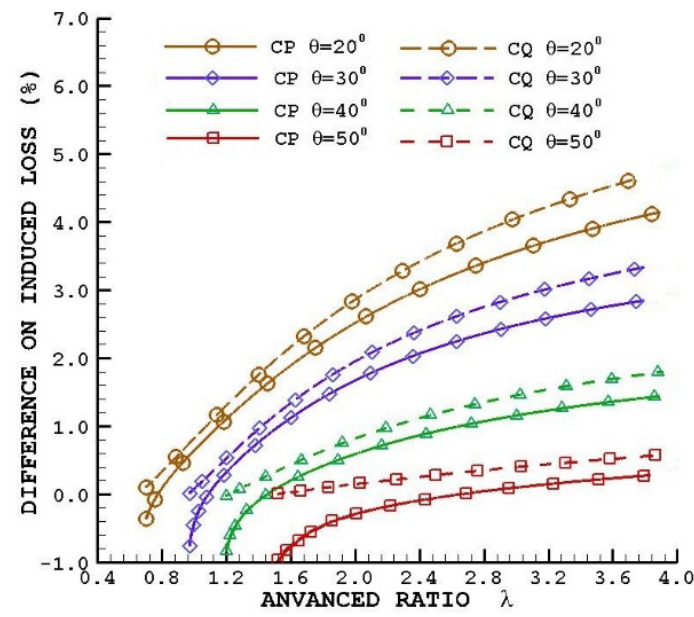

Fig. 3 Characteristic of tip induced loss of NACA six-blade single-rotating propeller yielding by current method

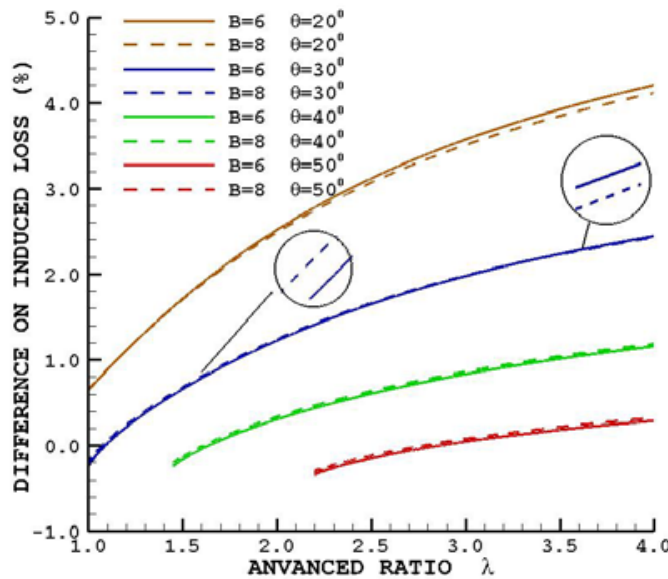

Fig. 4 Effect of the number of blades on the propeller-tip-induced power loss by current method

These two examples show that propeller with different geometry has different characteristics distribution of tip induced loss. The main difference lies in torque coefficient, which means the change of torque coefficient is not very big from and the torque coefficient almost has equal variation with the power coefficient from Figure 3. The reasons for this discrepancy are as follows. First of all, 
the advanced ratio of the variable propeller is larger than the fixed. Second, the number of rotor blades and the geometric parameters of the propeller are also different in two cases. Third, different performance on design point is also the reason for this discrepancy. For the loss characteristics induced by tip of these two instances, they show the same trend that loss improved with the increase of the distance ratio. There is only a difference in the size of the loss.

NACA did some experiments research on this variable pitch propeller with eight-blade single rotor or double rotor in history. In this paper, the research is more concerned in power loss. Here will be a further study on the influence of loss characteristics by blade numbers. The results are shown in Figure 4. For this single-rotor propeller, increasing the blades leads to some changes but not obvious. When the pitch angle is 20, the number of blades increasing cause the decrease of induced loss because high solidity leads to a negative angle of attack and then loss becomes large. When the pitch angle gradually rises to more than 30 , which tends to propeller design state, the increase of blades is caused by the increase of induced power loss. This phenomenon can be explained by the reason that increase of the loss is greater than the benefit from blade rectification. Based on the two trends above, blade number does not affect the magnitude of the tip induced loss in an intermediate pitch angle. It is 30 for this case. From another view, when pitch angle is fixed and number of blades is non-fixed, variation of advanced ratio can also result in the difference of the power loss. The characteristic shows the quite different tendencies by the number of blades due to the change of advanced ratio especially when pitch angle is 30 .

\section{Conclusion}

(1) The experimental data of loss characteristics is difficult to obtain because tip vortex is impossible to separate from 3D flow filed. But the method in this paper has considered the effect on the computational precision caused by compressibility and can be used for propeller design, especially in the high-density or low-speed conditions.

(2) For the different geometry of propeller, there is really some difference in characteristics distribution of tip induced loss. But the trend of power loss we care is consistent. When pitch angle is in fixed condition, loss rises with the increase of advanced ratio. When advanced is in given, induced loss decreases with larger pitch angle.

(3) The number of blades also has a certain impact on tip induced loss but not obvious, especially in the case with larger blade number. Furthermore, the influence of number on the characteristics of tip induced loss is often associated with specific pitch angle. The loss only from the effect about number of blades is different when this angle is different.

\section{Reference}

[1] Whitlow J B, Sievers G K. Fuel savings potential of the NASA Advanced Turboprop Program [M]. National Aeronautics and Space Administration, 1984.

[2] Dugan J F, Gatzen B S, Adamson W M. Prop-Fan Propulsion-Its Status and Potential[R]. SAE Technical Paper, 1978.

[3] Branlard E. Wind turbine tip-loss correction [D]. Master’s thesis, Risø DTU, Copenhagen, Denmark, 2013.

[4] Gur O, Rosen A. Comparison between blade-element models of propellers [J]. Aeronautical Journal, 2008, 112(1138): 689.

[5] Aviv R, Gur O. Propeller performance at low advance ratio [J]. Journal of Aircraft, 2005, 42(2): 435-441. 
[6] Crigler J L. Comparison of calculated and experimental propeller characteristics for four-, six-, and eight-blade single-rotating propellers [R]. National Advisory Committee for Aeronautics Langley Field va Langley Aeronautical Laboratory, 1944.

[7] Glauert H. Airplane propellers [M]//Aerodynamic theory. Springer Berlin Heidelberg, 1935: 169-360. 